\title{
VECTOR-VALUED SINGULAR INTEGRALS AND MAXIMAL FUNCTIONS ON SPACES OF HOMOGENEOUS TYPE
}

\author{
LOUKAS GRAFAKOS, LIGUANG LIU and DACHUN YANG*
}

\begin{abstract}
The Fefferman-Stein vector-valued maximal function inequality is proved for spaces of homogeneous type. The approach taken here is based on the theory of vector-valued Calderón-Zygmund singular integral theory in this context, which is appropriately developed.
\end{abstract}

\section{Introduction}

Let $(\mathscr{X}, d)$ be a metric space endowed with a regular Borel measure $\mu$. Recall that $\mu$ is said to be a regular Borel measure on $\mathscr{X}$ if $\mu$ is a nonnegative countably subadditive set function defined on all subsets of $\mathscr{X}$, open sets are measurable and every set is contained in a Borel set with the same measure (see, for example, [10]). We also assume that all balls defined by the metric $d$ have finite and positive measures on $\mu$. For any $x \in \mathscr{X}$ and $r>0$, set $B(x, r)=\{y \in$ $\mathscr{X}: d(x, y)<r\}$. The triple $(\mathscr{X}, d, \mu)$ is called a space of homogeneous type in the sense of Coifman and Weiss ([2], [3]) if the following doubling property holds: there exists a constant $C_{1} \geq 1$ such that for all $x \in \mathscr{X}$ and $r>0$,

$$
\mu(B(x, 2 r)) \leq C_{1} \mu(B(x, r)) .
$$

From (1.1), it is easy to deduce that there exist $n>0$ and $A \geq 1$ such that for all $x \in \mathscr{X}, r>0$ and $\lambda \geq 1$,

$$
\mu(B(x, \lambda r)) \leq A \lambda^{n} \mu(B(x, r)) .
$$

The number $n$ here in some sense measures the "dimension" of the space $\mathscr{X}$.

Let $\mathscr{B}$ be a complex Banach space with norm $\|\cdot\|_{\mathscr{B}}$. Let $\mathscr{B}^{*}$ be its dual space with norm $\|\cdot\|_{\mathscr{B}^{*}}$. A function $F$ defined on a $\sigma$-finite measure space $(\mathscr{X}, \mu)$

* The first author was supported by grant DMS 0400387 of the National Science Foundation of the USA and the University of Missouri Research Council. The third (corresponding) author was supported by the National Science Foundation for Distinguished Young Scholars (Grant No. 10425106), NCET (Grant No. 04-0142) of Ministry of Education of China.

Received September 10, 2007; in revised form November 30, 2007. 
and taking values in $\mathscr{B}$ is called $\mathscr{B}$-measurable if there exists a measurable subset $\mathscr{X}_{0}$ of $\mathscr{X}$ such that $\mu\left(\mathscr{X} \backslash \mathscr{X}_{0}\right)=0$ and $F\left(\mathscr{X}_{0}\right)$ is contained in some separable subspace $\mathscr{B}_{0}$ of $\mathscr{B}$, and for every $u^{*} \in \mathscr{B}^{*}$, the complex valued map $x \rightarrow\left\langle u^{*}, F(x)\right\rangle$ is measurable. From this definition and the theorem in [17, p. 131], it follows that the function $x \rightarrow\|F(x)\|_{\mathscr{B}}$ on $\mathscr{X}$ is measurable.

For any $p \in(0, \infty]$, define $L^{p}(\mathscr{X}, \mathscr{B})$ to be the space of all $\mathscr{B}$-measurable functions $F$ on $\mathscr{X}$ satisfying $\|F\|_{L^{p}(\mathscr{X}, \mathscr{B})}<\infty$, where

$$
\|F\|_{L^{p}(\mathscr{R}, \mathscr{B})}=\left\{\int_{\mathscr{L}}\|F(x)\|_{\mathscr{B}}^{p} d \mu(x)\right\}^{1 / p}
$$

with a usual modification made when $p=\infty$. Similarly, define $L^{p, \infty}(\mathscr{X}, \mathscr{B})$ to be the space of all $\mathscr{B}$-measurable functions $F$ on $\mathscr{X}$ satisfying $\|F\|_{L^{p, \infty}(\mathscr{X}, \mathscr{B})}$ $<\infty$, where

$$
\|F\|_{L^{p, \infty}(\mathscr{X}, \mathscr{B})}=\sup _{\alpha>0}\left\{\alpha\left[\mu\left(\left\{x \in \mathscr{X}:\|F(x)\|_{\mathscr{B}}>\alpha\right\}\right)\right]^{1 / p}\right\} .
$$

Let $p \in(0, \infty)$ and $L^{p}(\mathscr{X}) \otimes \mathscr{B}$ be the set of all finite linear combinations of elements of $\mathscr{B}$ with coefficients in $L^{p}(\mathscr{X})$, that is, elements of the form,

$$
F=f_{1} u_{1}+\cdots+f_{m} u_{m},
$$

where $m \in \mathrm{N}, f_{j} \in L^{p}(\mathscr{X})$ and $u_{j} \in \mathscr{B}$ for $j \in\{1, \ldots, m\}$. Then the space $L^{p}(\mathscr{X}) \otimes \mathscr{B}$ is dense in $L^{p}(\mathscr{X}, \mathscr{B})$; see, for example, [7] or Lemma 2.1 below. Given $F \in L^{1}(\mathscr{X}) \otimes \mathscr{B}$ as in (1.5), we define its integral to be the following element of $\mathscr{B}$

$$
\int_{\mathscr{X}} F(x) d \mu(x)=\sum_{j=1}^{m}\left\{\int_{\mathscr{L}} f_{j}(x) d \mu(x)\right\} u_{j} .
$$

Therefore, for any $F \in L^{1}(\mathscr{X}, \mathscr{B})$, the integral $\int_{\mathscr{L}} F(x) d \mu(x)$, as a unique extension of the integral of functions in $L^{1}(\mathscr{X}) \otimes \mathscr{B}$, is well defined; moreover, it is not difficult to show that for any $u^{*} \in \mathscr{B}^{*}$,

$$
\left\langle u^{*}, \int_{\mathscr{L}} F(x) d \mu(x)\right\rangle=\int_{\mathscr{L}}\left\langle u^{*}, F(x)\right\rangle d \mu(x),
$$

which further implies that

$$
\left\|\int_{\mathscr{L}} F(x) d \mu(x)\right\|_{\mathscr{B}} \leq \int_{\mathscr{L}}\|F(x)\|_{\mathscr{B}} d \mu(x)
$$

see, for example, [7] or [17] for more details. 
Now we turn to the vector-valued singular integrals. Let $\mathscr{B}_{1}$ and $\mathscr{B}_{2}$ be Banach spaces. Consider a kernel $\vec{K}$ defined on $(\mathscr{X} \times \mathscr{X}) \backslash \triangle$ with $\triangle=$ $\{(x, x): x \in \mathscr{X}\}$, where $\vec{K}(x, y)$ is an element of $\mathscr{L}\left(\mathscr{B}_{1}, \mathscr{B}_{2}\right)$, the space of all bounded linear operators from $\mathscr{B}_{1}$ to $\mathscr{B}_{2}$. The norm of $\vec{K}(x, y)$ will be denoted by $\|\vec{K}(x, y)\|_{\mathscr{B}_{1} \rightarrow \mathscr{B}_{2}}$.

Assume that $\vec{K}(x, y)$ is $\mathscr{L}\left(\mathscr{B}_{1}, \mathscr{B}_{2}\right)$-measurable and locally integrable on $(\mathscr{X} \times \mathscr{X}) \backslash \triangle$ such that the integral

$$
\vec{T}(F)(x)=\int_{\mathscr{X}} \vec{K}(x, y) F(y) d \mu(y)
$$

is well defined as an element of $\mathscr{B}_{2}$ for all $F \in L^{\infty}\left(\mathscr{X}, \mathscr{B}_{1}\right)$ with bounded support and $x \notin \operatorname{supp} F$. Assume that the kernel $\vec{K}$ satisfies Hörmander's conditions, i.e., there exists a positive constant $C_{H}$ such that for all $y, z \in \mathscr{X}$,

$$
\int_{d(x, y)>2 d(y, z)}\|\vec{K}(x, y)-\vec{K}(x, z)\|_{\mathscr{B}_{1} \rightarrow \mathscr{B}_{2}} d \mu(x) \leq C_{H},
$$

and for all $x, w \in \mathscr{X}$,

$$
\int_{d(x, y)>2 d(x, w)}\|\vec{K}(x, y)-\vec{K}(w, y)\|_{\mathscr{B}_{1} \rightarrow \mathscr{B}_{2}} d \mu(y) \leq C_{H} .
$$

The main result concerning such singular integrals is the following:

THEOREM 1.1. Let $\mathscr{B}_{1}$ and $\mathscr{B}_{2}$ be Banach spaces. Suppose that $\vec{T}$ given by (1.7) is a bounded linear operator from $L^{r}\left(\mathscr{X}, \mathscr{B}_{1}\right)$ to $L^{r}\left(\mathscr{X}, \mathscr{B}_{2}\right)$ for some $r \in$ $(1, \infty]$ with norm $A_{r}>0$. Assume that $\vec{K}$ satisfies Hörmander's conditions (1.8) and (1.9) for some $C_{H}>0$. Then $\vec{T}$ has well defined extensions on $L^{p}\left(\mathscr{X}, \mathscr{B}_{1}\right)$ for all $p \in[1, \infty)$. Moreover, there exist positive constants $C_{\mathscr{X}}$ and $\widetilde{C}_{\mathscr{X}}$ depending only on $\mathscr{X}$ such that for all $F \in L^{1}\left(\mathscr{X}, \mathscr{B}_{1}\right)$,

$$
\|\vec{T}(F)\|_{L^{1, \infty}\left(\mathscr{X}, \mathscr{B}_{2}\right)} \leq \widetilde{C}_{\mathscr{X}}\left(C_{H}+A_{r}\right)\|F\|_{L^{1}\left(\mathscr{X}, \mathscr{B}_{1}\right)},
$$

and whenever $p \in(1, \infty)$, for all $F \in L^{p}\left(\mathscr{X}, \mathscr{B}_{1}\right)$,

$$
\|\vec{T}(F)\|_{L^{p}\left(\mathscr{X}, \mathscr{B}_{2}\right)} \leq C_{\mathscr{X}} C_{p}\left(C_{H}+A_{r}\right)\|F\|_{L^{p}\left(\mathscr{X}, \mathscr{B}_{1}\right)},
$$

where $C_{p}=\max \left\{p,(p-1)^{-1}\right\}$. When $r=\infty$, (1.11) holds with $C_{p}=$ $\max \left\{1,(p-1)^{-1}\right\}$.

For any $f \in L_{\text {loc }}^{1}(\mathscr{X})$ and $x \in \mathscr{X}$, the Hardy-Littlewood maximal function $\mathcal{M}(f)$ is defined by

$$
\mathscr{M}(f)(x)=\sup _{B \ni x} \frac{1}{\mu(B)} \int_{B}|f(y)| d \mu(y),
$$


where the supremum is taken over all balls $B$ containing $x$, and the central Hardy-Littlewood maximal function $\widetilde{\mathscr{M}}(f)$ is defined by

$$
\tilde{M}(f)(x)=\sup _{r>0} \frac{1}{\mu(B(x, r))} \int_{B(x, r)}|f(y)| d \mu(y) .
$$

Then it is easy to deduce that $\tilde{\mathscr{M}}(f)(x) \leq \mathscr{M}(f)(x) \leq A 3^{n} \tilde{\mathscr{M}}(f)(x)$ for all $x \in \mathscr{X}$. Moreover, $\mathscr{M}$ is weak-type $(1,1)$ and bounded on $L^{p}(\mathscr{X})$ for $p \in(1, \infty]$; see [2], [3] for more details.

As an application of Theorem 1.1, we obtain the Fefferman-Stein vectorvalued maximal function inequality first proved by [5] for Euclidean spaces; see also [14].

Theorem 1.2. Let $M$ be the Hardy-Littlewood maximal operator. For $p \in$ $(1, \infty)$ and $q \in(1, \infty]$, there exist positive constants $C_{\mathscr{L}}$ and $\widetilde{C}_{\mathscr{L}}$ depending only on $\mathscr{X}$ such that for all measurable functions $\left\{f_{j}\right\}_{j \in \mathrm{N}}$,

$$
\left\|\left(\sum_{j \in \mathrm{N}}\left[\mathcal{M}\left(f_{j}\right)\right]^{q}\right)^{1 / q}\right\|_{\left.L^{1, \infty} \mathscr{X}\right)} \leq \widetilde{C}_{\mathscr{X}} C_{q}\left\|\left(\sum_{j \in \mathrm{N}}\left|f_{j}\right|^{q}\right)^{1 / q}\right\|_{L^{1}(\mathscr{C})}
$$

and

$$
\left\|\left(\sum_{j \in \mathrm{N}}\left[\mathcal{M}\left(f_{j}\right)\right]^{q}\right)^{1 / q}\right\|_{L^{p}(\mathscr{X})} \leq C_{\mathscr{X}} C_{p, q}\left\|\left(\sum_{j \in \mathrm{N}}\left|f_{j}\right|^{q}\right)^{1 / q}\right\|_{L^{p}(\mathscr{X})},
$$

where $C_{q}=\max \left\{1,(q-1)^{-1}\right\}$ and $C_{p, q}=\max \left\{p,(p-1)^{-1}\right\} \max \{1,(q-$ $\left.1)^{-1}\right\}$ if $q \in(1, \infty)$; and if $q=\infty, C_{q}$ and $C_{p, q}$ coincide with the norms of the operator $\mathscr{M}: L^{1}(\mathscr{X}) \rightarrow L^{1, \infty}(\mathscr{X})$ and $\mathscr{M}: L^{p}(\mathscr{X}) \rightarrow L^{p}(\mathscr{X})$, respectively.

Theorems 1.1 and 1.2 are classical and well known in the Euclidean setting. Their extension to spaces of homogeneous type is dictated by the wide range of applications in which they appear; see for instance [13], [8], [9], [12] for applications involving function spaces and [13] for an application in the context of smooth manifolds with geometry given by a Carnot-Carathéodory metric induced by a collection of vector fields of finite type.

One may give another proof of Theorem 1.2 using the weighted theory on spaces of homogeneous type (see, for instance, [16]) and by a procedure as in [5] (see also [14, Chapter II]). This was pointed out in [13] but neither that proof nor the one in this paper has been previously published in this general framework with careful verification of all the details involved, some of which are rather delicate. 
The organization of the paper is as follows. In Section 2, we discuss a suitable adaptation of the Calderón-Zygmund decomposition on $\mathscr{X}$ to the vectorvalued setting and we prove Theorem 1.1 following [6, Section V.3] (see also [7, pp. 326-327]). The proof of Theorem 1.2 is given in Section 3 and is also based on the approach in [6, Section V.4].

We use the following notation: $\mathbf{N}=\{1,2, \ldots\}, \mathbf{Z}_{+}=\mathbf{N} \cup\{0\}$, and for $p \in[1, \infty], p^{\prime}$ is the unique element of $[1, \infty]$ satisfying $1 / p+1 / p^{\prime}=1$. We denote by $C$ positive constants independent of the main parameters involved, which may vary at different occurrences. Constants with subscripts do not change through the whole paper. We use $f \lesssim g$ and $f \gtrsim g$ to denote $f \leq C g$ and $f \geq C g$, respectively. If $f \lesssim g \lesssim f$, we then write $f \sim g$. For any $x \in \mathscr{X}$ and $r>0$, denote $\mu(B(x, r))$ by $V_{r}(x)$.

\section{Proof of Theorem 1.1}

We begin with the following density lemma, parts of whose conclusions are already known and others are easy. We omit the details; see, for example, [7, pp. 320-321] or [4].

Lemma 2.1. For $p \in(0, \infty)$, the set of functions with the form

$$
\Phi(x)=\sum_{j=1}^{m} \chi_{E_{j}}(x) u_{j}
$$

where $m \in \mathrm{N}, u_{j} \in \mathscr{B},\left\{E_{j}\right\}_{j=1}^{m}$ are pairwise disjoint bounded subsets of $\mathscr{X}$ and $0<\mu\left(E_{j}\right)<\infty$, is dense in $L^{p}(\mathscr{X}, \mathscr{B})$. For $p=\infty$, the set of functions with the form $\Phi(x)=\sum_{j=1}^{\infty} \chi_{E_{j}}(x) u_{j}$, where $\left\{u_{j}\right\}_{j \in \mathrm{N}} \subset \mathscr{B}$ and $\left\{E_{j}\right\}_{j \in \mathrm{N}}$ is a partition of $\mathscr{X}$, is dense in $L^{\infty}(\mathscr{X}, \mathscr{B})$.

Remark 2.2. Denote by $L_{b}^{\infty}(\mathscr{X}, \mathscr{B})$ the set of functions in $L^{\infty}(\mathscr{X}, \mathscr{B})$ with bounded support. For $p \in(0, \infty)$, Lemma 2.1 says that $L^{p}(\mathscr{X}) \otimes \mathscr{B}$ is dense in $L^{p}(\mathscr{X}, \mathscr{B})$, which further implies that $L_{b}^{\infty}(\mathscr{X}, \mathscr{B})$ is also dense in $L^{p}(\mathscr{X}, \mathscr{B})$.

Using Lemma 2.1 and arguing as in [4, pp. 97-98], we obtain the following conclusions by leaving the details to the reader. By $\mathscr{B}^{*}$ we denote the dual space of $\mathscr{B}$.

Lemma 2.3. Let $p \in[1, \infty], p^{\prime}$ be the conjugate index of $p$, i.e., $1 / p+$ $1 / p^{\prime}=1$ and let $\mathscr{B}$ be a Banach space. Then,

(a) for any $F \in L^{p}(\mathscr{X}, \mathscr{B})$,

$$
\|F\|_{L^{p}(\mathscr{X}, \mathscr{B})}=\sup _{\|G\|_{L^{p^{\prime}}\left(\mathscr{X}, \mathscr{B}^{*}\right)} \leq 1}\left|\int_{\mathscr{L}}\langle G(x), F(x)\rangle d \mu(x)\right| ;
$$


(b) for any $G \in L^{p^{\prime}}\left(\mathscr{X}, \mathscr{B}^{*}\right)$,

$$
\|G\|_{L^{p^{\prime}}\left(\mathscr{X}, \mathscr{B}^{*}\right)}=\sup _{\|F\|_{L^{p}(\mathscr{X}, \mathscr{B})} \leq 1}\left|\int_{\mathscr{X}}\langle G(x), F(x)\rangle d \mu(x)\right| .
$$

REMARK 2.4. From Theorem 1 in [4, p. 98] and Example 1 in [4, p. 60], it follows that in general, $L^{p^{\prime}}\left(\mathscr{X}, \mathscr{B}^{*}\right) \subsetneq\left(L^{p}(\mathscr{X}, \mathscr{B})\right)^{*}$ for $p \in[1, \infty)$. However, if $\mathscr{B}$ is reflexive and separable, then $L^{p^{\prime}}\left(\mathscr{X}, \mathscr{B}^{*}\right)=\left(L^{p}(\mathscr{X}, \mathscr{B})\right)^{*}$ by Corollary 4 in [4, p. 82] and Theorem 1 in [4, p. 98].

The following vector-valued version of the Marcinkiewicz interpolation is essentially contained in [1, Lemma 1]. The formulation below keeps into account the constants; the details of the proof are standard and omitted.

Lemma 2.5. Let $\mathscr{B}_{1}$ and $\mathscr{B}_{2}$ be Banach spaces. Let $0<p_{0} \leq p<p_{1} \leq \infty$ and $s \in(0,1)$ satisfy $(1-s) / p_{0}+s / p_{1}=1 / p$. Suppose that $\vec{T}$ is a sublinear operator, that is, it satisfies

$$
\|\vec{T}(F+G)\|_{\mathscr{B}_{2}} \leq\|\vec{T}(F)\|_{\mathscr{B}_{2}}+\|\vec{T}(G)\|_{\mathscr{B}_{2}}
$$

for all $F$ and $G$. Assume that $\vec{T}$ maps $L^{p_{0}}\left(\mathscr{X}, \mathscr{B}_{1}\right)$ to $L^{p_{0}, \infty}\left(\mathscr{X}, \mathscr{B}_{2}\right)$ with norm $A_{0}$ and $L^{p_{1}}\left(\mathscr{X}, \mathscr{B}_{1}\right)$ to $L^{p_{1}, \infty}\left(\mathscr{X}, \mathscr{B}_{2}\right)$ with norm $A_{1}$. Then $\vec{T}$ maps $L^{p}\left(\mathscr{X}, \mathscr{B}_{1}\right)$ to $L^{p}\left(\mathscr{X}, \mathscr{B}_{2}\right)$ with norm at most $2\left(\frac{p}{p-p_{0}}+\frac{p}{p_{1}-p}\right)^{1 / p} A_{0}^{1-s} A_{1}^{s}$.

Using Lemma 2.1 and an adaptation of the proof of the classical RieszThorin interpolation theorem (see, for example, [7], [15]), we obtain the following result. The details are omitted.

Lemma 2.6. Let $\mathscr{B}_{1}$ and $\mathscr{B}_{2}$ be Banach spaces. Let $1 \leq p_{0}, p_{1}, q_{0}, q_{1} \leq \infty$, $s \in(0,1), 1 / p=(1-s) / p_{0}+s / p_{1}$ and $1 / q=(1-s) / q_{0}+s / q_{1}$. Assume that $\vec{T}$ is a bounded linear operator from $L^{p_{i}}\left(\mathscr{X}, \mathscr{B}_{1}\right)$ to $L^{q_{i}}\left(\mathscr{X}, \mathscr{B}_{2}\right)$ with norm $A_{i}$, where $i=1,2$. Then $\vec{T}$ is bounded from $L^{p}\left(\mathscr{X}, \mathscr{B}_{1}\right)$ to $L^{q}\left(\mathscr{X}, \mathscr{B}_{2}\right)$ with norm at most $A_{0}^{1-s} A_{1}^{s}$.

From Lemma 2.5 and Lemma 2.6, we deduce the following conclusion; see [7, p. 43] for the scalar case.

Corollary 2.7. Let $\mathscr{B}_{1}$ and $\mathscr{B}_{2}$ be Banach spaces. Let $1<p<r \leq \infty$. Suppose that $\vec{T}$ is a linear operator bounded from $L^{1}\left(\mathscr{X}, \mathscr{B}_{1}\right)$ to $L^{1, \infty}\left(\mathscr{X}, \mathscr{B}_{2}\right)$ with norm $A_{0}$ and from $L^{r}\left(\mathscr{X}, \mathscr{B}_{1}\right)$ to $L^{r}\left(\mathscr{X}, \mathscr{B}_{2}\right)$ with norm $A_{1}$. Then $\vec{T}$ is bounded from $L^{p}\left(\mathscr{X}, \mathscr{B}_{1}\right)$ to $L^{p}\left(\mathscr{X}, \mathscr{B}_{2}\right)$ with norm at most $8 \max \{1,(p-$ $\left.1)^{-1}\right\}^{1 / p} A_{0}^{\frac{1 / p-1 / r}{1-1 / r}} A_{1}^{\frac{1-1 / p}{1-1 / r}}$.

Proof. Applying Lemma 2.5 and interpolating between $L^{1}\left(\mathscr{X}, \mathscr{B}_{1}\right)$ and $L^{r}\left(\mathscr{X}, \mathscr{B}_{1}\right)$, we obtain the boundedness of $\vec{T}$ from $L^{(p+1) / 2}\left(\mathscr{X}, \mathscr{B}_{1}\right)$ to 
$L^{(p+1) / 2}\left(\mathscr{X}, \mathscr{B}_{2}\right)$. Then using Lemma 2.6 and interpolating between $L^{(p+1) / 2}\left(\mathscr{X}, \mathscr{B}_{1}\right)$ and $L^{r}\left(\mathscr{X}, \mathscr{B}_{1}\right)$, we further obtain the boundedness of $\vec{T}$ from $L^{p}\left(\mathscr{X}, \mathscr{B}_{1}\right)$ to $L^{p}\left(\mathscr{X}, \mathscr{B}_{2}\right)$, which completes the proof of Corollary 2.7.

The Calderón-Zygmund decomposition in Coifman and Weiss [2] can be generalized to functions in $L^{1}(\mathscr{X}, \mathscr{B})$ with bounded support as follows.

Lemma 2.8. Let $F \in L^{1}(\mathscr{X}, \mathscr{B})$ with bounded support and $\alpha>$ $\|F\|_{L^{1}(\mathscr{L}, \mathscr{B})} / \mu(\mathscr{X})$. Then there exist positive constants $C_{2}, M$ depending only on $\mathscr{X}$ and a sequence of metric balls $\left\{B\left(x_{i}, r_{i}\right)\right\}_{i}$ such that

(i) $F(x)=g(x)+h(x)$, where $h(x)=\sum_{i} h_{i}(x)$ holds for all $x \in \mathscr{X}$;

(ii) for almost every $x \in \mathscr{X},\|g(x)\|_{\mathscr{B}} \leq C_{2} \alpha$;

(iii) $\|g\|_{L^{1}(\mathscr{L}, \mathscr{B})} \leq C_{2}\|F\|_{L^{1}(\mathscr{L}, \mathscr{B})}$;

(iv) for any $i$, supp $h_{i} \subset B\left(x_{i}, r_{i}\right)$ and $\sum_{i} \mu\left(B\left(x_{i}, r_{i}\right)\right) \leq C_{2}\|F\|_{L^{1}(\mathscr{X}, \mathscr{B})} / \alpha$;

(v) for any $i, \int_{\mathscr{L}} h_{i}(x) d \mu(x)=\theta_{\mathscr{B}}$, where $\theta_{\mathscr{B}}$ denotes the zero element of $\mathscr{B}$;

(vi) $\sum_{i}\left\|h_{i}\right\|_{L^{1}(\mathscr{C}, \mathscr{B})} \leq C_{2}\|F\|_{L^{1}(\mathscr{L}, \mathscr{B})}$;

(vii) every point of $\mathscr{X}$ belongs to no more than $M$ balls of $\left\{B\left(x_{i}, r_{i}\right)\right\}_{i}$.

Proof. We only give a sketch of the proof. Given any $\alpha>0$, set

$$
\Omega_{\alpha}=\left\{x \in \mathscr{X}: \mathscr{M}\left(\|F(\cdot)\|_{\mathscr{B}}\right)(x)>A^{2} 6^{n} \alpha\right\} .
$$

Then the weak- $(1,1)$ property of $\mathscr{M}$ implies that $\mu\left(\Omega_{\alpha}\right) \lesssim\|F\|_{L^{1}(\mathscr{L}, \mathscr{B})} / \alpha<$ $\infty$. It is obvious that $\Omega_{\alpha}$ is open. By [3, Lemma (3.9)], we know that $\Omega_{\alpha}$ is bounded, i.e., $\Omega_{\alpha}$ is contained in some ball of $\mathscr{X}$.

Thus, applying the Whitney covering lemma (see [2], [3]) yields a sequence of balls $\left\{B\left(x_{i}, r_{i}\right)\right\}_{i}$ satisfying that: (1) $\Omega_{\alpha}=\bigcup_{i} B\left(x_{i}, r_{i}\right)$; (2) every point $x \in \mathscr{X}$ belongs to no more than $M$ balls of $\left\{B\left(x_{i}, r_{i}\right)\right\}_{i}$; (3) there exists a constant $C>1$ depending only on $\mathscr{X}$ such that $\left\{B\left(x_{i}, C^{-1} r_{i}\right)\right\}_{i}$ are mutually disjoint and $B\left(x_{i}, C r_{i}\right) \cap \Omega_{\alpha}^{\complement} \neq \varnothing$ for any $i$, where and in what follows, $\Omega_{\alpha}^{\complement}=\mathscr{X} \backslash \Omega_{\alpha}$.

For any $i$, set $B_{i}=B\left(x_{i}, r_{i}\right)$ and

$$
\eta_{i}(x)=\frac{\chi_{B_{i}}(x)}{\sum_{j} \chi_{B_{j}}(x)},
$$

where $\chi_{B_{i}}(x)=1$ if $x \in B_{i}$, and $=0$ if $x \notin B_{i}$. Define

$$
g(x)=F(x) \chi_{\Omega_{\alpha}^{\mathrm{C}}}(x)+\sum_{i}\left(\frac{1}{\mu\left(B_{i}\right)} \int_{B_{i}} F(y) \eta_{i}(y) d \mu(y)\right) \chi_{B_{i}}(x),
$$


and for any given $i$,

$$
h_{i}(x)=F(x) \eta_{i}(x)-\left(\frac{1}{\mu\left(B_{i}\right)} \int_{B_{i}} F(y) \eta_{i}(y) d \mu(y)\right) \chi_{B_{i}}(x) .
$$

Notice that $F(y) \eta_{i}(y)$ is $\mathscr{B}$-measurable and the integral $\int_{B_{i}} F(y) \eta_{i}(y) d \mu(y)$ makes sense, which imply that $g$ and $h_{i}$ are well defined. Properties (i) through (vii) above can then be verified easily. This completes the proof of Lemma 2.8.

Proof of Theorem 1.1. Since $L_{b}^{\infty}\left(\mathscr{X}, \mathscr{B}_{1}\right)$ is dense in $L^{p}\left(\mathscr{X}, \mathscr{B}_{1}\right)$ for $p \in[1, \infty)$ (see Remark 2.2), we only need to verify the theorem for $F \in$ $L_{b}^{\infty}\left(\mathscr{X}, \mathscr{B}_{1}\right)$. We further assume that $\mu(\mathscr{X})<\infty$ since the proof for the case $\mu(\mathscr{X})=\infty$ is similar and simple.

We first show the theorem for the case $r<\infty$. Let us now prove the weak estimate (1.10). If $0<A_{r}^{-1} \lambda \leq\|F\|_{L^{1}(\mathscr{C}, \mathscr{B})} / \mu(\mathscr{X})$ (this happens only when $\mu(\mathscr{X})<\infty)$, then

$$
\mu\left(\left\{x \in \mathscr{X}:\|\vec{T}(F)(x)\|_{\mathscr{B}_{2}}>\lambda\right\}\right) \leq \mu(\mathscr{X}) \leq A_{r} \frac{\|F\|_{L^{1}\left(\mathscr{X}, \mathscr{B}_{1}\right)}}{\lambda} .
$$

If $A_{r}^{-1} \lambda>\|F\|_{L^{1}\left(\mathscr{X}, \mathscr{B}_{1}\right)} / \mu(\mathscr{X})$, then applying Lemma 2.8 to $F \in L_{b}^{\infty}\left(\mathscr{X}, \mathscr{B}_{1}\right)$ and $A_{r}^{-1} \lambda$ yields that

$$
F(x)=g(x)+h(x)=g(x)+\sum_{i} h_{i}(x),
$$

where $g$ and $h_{i}$ satisfy Properties (i) through (vii) of Lemma 2.8 with constant $\alpha$ replaced by $A_{r}^{-1} \lambda$. For any $i$, set $\widetilde{B}_{i}=B\left(x_{i}, 2 r_{i}\right)$. Notice that

$$
\begin{aligned}
\mu\left(\left\{x \in \mathscr{X}:\|\vec{T}(F)(x)\|_{\mathscr{B}_{2}}>\lambda\right\}\right) \\
\leq \mu\left(\left\{x \in \mathscr{X}:\|\vec{T}(g)(x)\|_{\mathscr{B}_{2}}>\lambda / 2\right\}\right) \\
\quad+\mu\left(\left\{x \in \mathscr{X}:\|\vec{T}(h)(x)\|_{\mathscr{B}_{2}}>\lambda / 2\right\}\right) \\
\leq \frac{2^{r}}{\lambda^{r}}\|\| \vec{T}(g)\left\|_{\mathscr{B}_{2}}\right\|_{L^{r}(\mathscr{C})}^{r}+\mu\left(\bigcup_{j} \widetilde{B}_{j}\right) \\
\quad+\mu\left(\left\{x \notin \bigcup_{j} \widetilde{B}_{j}:\|\vec{T}(h)(x)\|_{\mathscr{B}_{2}}>\lambda / 2\right\}\right) \\
\equiv \mathrm{Z}_{1}+\mathrm{Z}_{2}+\mathrm{Z}_{3} .
\end{aligned}
$$

The boundedness of $\vec{T}$ from $L^{r}\left(\mathscr{X}, \mathscr{B}_{1}\right)$ to $L^{r}\left(\mathscr{X}, \mathscr{B}_{2}\right)$ together with Lemma 2.8 (ii) and (iii) shows that

$$
\mathrm{Z}_{1} \leq \frac{\left(2 A_{r}\right)^{r}}{\lambda^{r}}\|g\|_{L^{r}\left(\mathscr{L}, \mathscr{B}_{1}\right)}^{r} \leq \frac{\left(2 C_{2}\right)^{r} A_{r}}{C_{2} \lambda}\|g\|_{L^{1}\left(\mathscr{L}, \mathscr{B}_{1}\right)} \leq \frac{\left(2 C_{2}\right)^{r} A_{r}}{\lambda}\|F\|_{L^{1}\left(\mathscr{X}, \mathscr{B}_{1}\right)} .
$$


By (1.2) and Lemma 2.8 (iv),

$$
\mathrm{Z}_{2} \leq A 2^{n} \sum_{j} \mu\left(B\left(x_{j}, r_{j}\right)\right) \leq \frac{A 2^{n} C_{2} A_{r}}{\lambda}\|F\|_{L^{1}\left(\mathscr{X}, \mathscr{B}_{1}\right)} .
$$

Now we estimate $Z_{3}$. Notice that Lemma 2.8 (v) and (1.6) imply that for any $x \notin\left(\bigcup_{j} \widetilde{B}_{j}\right)$,

$$
\begin{aligned}
\|\vec{T}(h)(x)\|_{\mathscr{B}_{2}} & =\left\|\sum_{j} \int_{B\left(x_{j}, r_{j}\right)}\left[\vec{K}(x, y)-\vec{K}\left(x, x_{j}\right)\right] h_{j}(y) d \mu(y)\right\|_{\mathscr{B}_{2}} \\
& \leq \sum_{j} \int_{B\left(x_{j}, r_{j}\right)}\left\|\left[\vec{K}(x, y)-\vec{K}\left(x, x_{j}\right)\right] h_{j}(y)\right\|_{\mathscr{B}_{2}} d \mu(y) \\
& \leq \sum_{j} \int_{B\left(x_{j}, r_{j}\right)}\left\|\vec{K}(x, y)-\vec{K}\left(x, x_{j}\right)\right\|_{\mathscr{B}_{1} \rightarrow \mathscr{B}_{2}}\left\|h_{j}(y)\right\|_{\mathscr{B}_{1}} d \mu(y) .
\end{aligned}
$$

Thus,

$$
\begin{aligned}
& \mathrm{Z}_{3} \\
& \leq \frac{2}{\lambda} \int_{\left(\bigcup_{j} \widetilde{B}_{j}\right)^{\complement}}\|\vec{T}(h)(x)\|_{\mathscr{B}_{2}} d \mu(x) \\
& \leq \frac{2}{\lambda} \sum_{j} \int_{B\left(x_{j}, r_{j}\right)} \int_{\left(\bigcup_{j} \widetilde{B}_{j}\right)^{\complement}}\left\|\vec{K}(x, y)-\vec{K}\left(x, x_{j}\right)\right\|_{\mathscr{B}_{1} \rightarrow \mathscr{B}_{2}}\left\|h_{j}(y)\right\|_{\mathscr{B}_{1}} d \mu(x) d \mu(y) .
\end{aligned}
$$

For any $j$, since $x \notin\left(\bigcup_{j} \widetilde{B}_{j}\right)$ implies that $x \notin \widetilde{B}_{j}$, it follows that for any $y \in B\left(x_{j}, r_{j}\right), d\left(x, x_{j}\right) \geq 2 r_{j}>2 d\left(y, x_{j}\right)$. Then by (1.8) and Lemma 2.8 (vi),

$$
\mathrm{Z}_{3} \leq \frac{2 C_{H}}{\lambda} \sum_{j} \int_{B\left(x_{j}, r_{j}\right)}\left\|h_{j}(y)\right\|_{\mathscr{B}_{1}} d \mu(y) \leq \frac{2 C_{H} C_{2}}{\lambda}\|F\|_{L^{1}\left(\mathscr{X}, \mathscr{B}_{1}\right)} .
$$

Then combining (2.1) and the estimates of $Z_{1}$ through $Z_{3}$ yields (1.10) for the case $r<\infty$.

By Corollary 2.7 and interpolating between $\vec{T}: L^{1}\left(\mathscr{X}, \mathscr{B}_{1}\right) \rightarrow L^{1, \infty}\left(\mathscr{X}, \mathscr{B}_{2}\right)$ and $\vec{T}: L^{r}\left(\mathscr{X}, \mathscr{B}_{1}\right) \rightarrow L^{r}\left(\mathscr{X}, \mathscr{B}_{2}\right)$, we then have that for $p \in(1, r)$, there exists a constant $C_{\mathscr{L}}>0$ such that for all $F \in L^{p}\left(\mathscr{X}, \mathscr{B}_{1}\right)$,

$$
\|\vec{T}(F)\|_{L^{p}\left(\mathscr{X}, \mathscr{B}_{2}\right)} \leq C_{\mathscr{X}} \max \left\{1,(p-1)^{-1}\right\}\left(C_{H}+A_{r}\right)\|F\|_{L^{p}\left(\mathscr{X}, \mathscr{B}_{1}\right)},
$$

namely, (1.11) holds for $p \in(1, r)$.

We still need to verify (1.11) for $p>r$. From $\vec{K} \in \mathscr{L}\left(\mathscr{B}_{1}, \mathscr{B}_{2}\right)$, it follows that its adjoint $\vec{K}^{*} \in \mathscr{L}\left(\mathscr{B}_{2}^{*}, \mathscr{B}_{1}^{*}\right)$ has the same norm as $\vec{K}$. Therefore, (1.9) 
for $\vec{K}$ is equivalent to (1.8) for $\vec{K}^{*}$. The boundedness of $\vec{T}$ from $L^{r}\left(\mathscr{X}, \mathscr{B}_{1}\right)$ to $L^{r}\left(\mathscr{X}, \mathscr{B}_{2}\right)$ and Lemma $2.3(b)$ together with Hölder's inequality show that for any $F \in L^{r^{\prime}}\left(\mathscr{X}, \mathscr{B}_{2}^{*}\right)$,

$$
\begin{aligned}
& \left\|\vec{T}^{*}(F)\right\|_{L^{r^{\prime}}\left(\mathscr{L}, \mathscr{B}_{1}^{*}\right)}=\sup _{\|G\|_{L^{r}\left(\mathscr{X}, \mathscr{P}_{1}\right)} \leq 1}\left|\int_{\mathscr{L}}\left\langle\vec{T}^{*}(F)(x), G(x)\right\rangle d \mu(x)\right| \\
& \leq \sup _{\|G\|_{L^{r}\left(\mathscr{X}, \mathscr{B}_{1}\right)} \leq 1} \int_{\mathscr{X}}\|F(x)\|_{\mathscr{B}_{2}^{*}}\|\vec{T}(G)(x)\|_{\mathscr{B}_{2}} d \mu(x) \\
& \leq \sup _{\|G\|_{L^{r}\left(\mathscr{Q}, \mathscr{B}_{1}\right)} \leq 1}\|F\|_{L^{r^{\prime}}\left(\mathscr{X}, \mathscr{B}_{2}^{*}\right)}\|\vec{T}(G)\|_{L^{r}\left(\mathscr{X}, \mathscr{B}_{2}\right)} \\
& \leq A_{r}\|F\|_{L^{r^{\prime}\left(\mathscr{X}, \mathscr{B}_{2}^{*}\right)}} \text {. }
\end{aligned}
$$

That is, $\vec{T}^{*}$ is bounded from $L^{r^{\prime}}\left(\mathscr{X}, \mathscr{B}_{2}^{*}\right)$ to $L^{r^{\prime}}\left(\mathscr{X}, \mathscr{B}_{1}^{*}\right)$ with norm at most $A_{r}$. Repeating the proof above for (1.10), we obtain

$$
\left\|\vec{T}^{*}(F)\right\|_{L^{1, \infty}\left(\mathscr{R}, \mathscr{B}_{1}^{*}\right)} \leq C_{\mathscr{X}}\left(C_{H}+A_{r}\right)\|F\|_{L^{1}\left(\mathscr{L}, \mathscr{B}_{2}^{*}\right)} .
$$

Then using these bounds and interpolating yield that for any $p \in\left(1, r^{\prime}\right)$,

$$
\left\|\vec{T}^{*}(F)\right\|_{L^{p^{\prime}}\left(\mathscr{X}, \mathscr{B}_{1}^{*}\right)} \leq C_{\mathscr{L}} \max \{1, p-1\}\left(C_{H}+A_{r}\right)\|F\|_{L^{p^{\prime}}\left(\mathscr{C}, \mathscr{B}_{2}^{*}\right)},
$$

since $\left(p^{\prime}-1\right)^{-1}=p-1$. Hence, (2.3) and Lemma $2.3(a)$ together with Hölder's inequality give that for any $p \in(r, \infty)$,

$$
\begin{aligned}
\|\vec{T}(F)\|_{L^{p}\left(\mathscr{X}, \mathscr{B}_{2}\right)} & =\sup _{\|G\|_{L^{p^{\prime}}\left(\mathscr{X}, \mathscr{O}_{2}^{*}\right)} \leq 1}\left|\int_{\mathscr{X}}\langle G(x), \vec{T}(F)(x)\rangle d \mu(x)\right| \\
& \leq \sup _{\|G\|_{L^{p^{\prime}}\left(\mathscr{X}, \mathscr{O}_{2}^{*}\right)} \leq 1}\left\|\vec{T}^{*}(G)\right\|_{L^{p^{\prime}}\left(\mathscr{X}, \mathscr{B}_{1}^{*}\right)}\|F\|_{L^{p}\left(\mathscr{X}, \mathscr{B}_{1}\right)} \\
& \leq C_{\mathscr{X}} \max \{1, p-1\}\left(C_{H}+A_{r}\right)\|F\|_{L^{p}\left(\mathscr{X}, \mathscr{B}_{1}\right)} .
\end{aligned}
$$

This estimate combined with (2.2) yields (1.11). Thus Theorem 1.1 holds when $r<\infty$.

Now we consider the case $r=\infty$. Notice that if

$$
0<\lambda \leq 2 A_{\infty} C_{2}\|F\|_{L^{1}(\mathscr{X}, \mathscr{B})} / \mu(\mathscr{X}),
$$

then in a way similar to the case $r<\infty$, we obtain (2.1) with $A_{r}$ replaced by $2 A_{\infty} C_{2}$. Otherwise, we apply Lemma 2.8 to $F \in L_{b}^{\infty}\left(\mathscr{X}, \mathscr{B}_{1}\right)$ and $\left(2 A_{\infty} C_{2}\right)^{-1} \lambda>\|F\|_{L^{1}(\mathscr{L}, \mathscr{B})} / \mu(\mathscr{X})$. Moreover, since for any $x \in \mathscr{X}$, the 
boundedness of $\vec{T}$ from $L^{\infty}\left(\mathscr{X}, \mathscr{B}_{1}\right)$ to $L^{\infty}\left(\mathscr{X}, \mathscr{B}_{2}\right)$ and Lemma 2.8 (ii) imply that for all $x \in \mathscr{X}$,

$$
\|\vec{T}(g)(x)\|_{\mathscr{B}_{2}} \leq A_{\infty}\|g(x)\|_{\mathscr{B}_{1}} \leq \lambda / 2
$$

we then have

$$
\mu\left(\left\{x \in \mathscr{X}:\|\vec{T}(F)(x)\|_{\mathscr{B}_{2}}>\lambda\right\}\right) \leq \mu\left(\left\{x \in \mathscr{X}:\|\vec{T}(h)(x)\|_{\mathscr{B}_{2}}>\lambda / 2\right\}\right) .
$$

Then repeating the estimates above for $Z_{2}$ and $Z_{3}$ yields (1.10), which together with Corollary 2.7 further shows that $(2.2)$ holds for any $p \in(1, \infty)$. This finishes the proof of Theorem 1.1.

By Theorem 1.1 and an argument similar to the proof of [7, Proposition 4.6.4], we obtain the following conclusion, whose details are left to the reader.

Corollary 2.9. Let $p, q \in(1, \infty)$ and $\mathscr{B}_{1}, \mathscr{B}_{2}$ be Banach spaces. Suppose that $\vec{T}$ given by (1.7) is a bounded linear operator from $L^{q}\left(\mathscr{X}, \mathscr{B}_{1}\right)$ to $L^{q}\left(\mathscr{X}, \mathscr{B}_{2}\right)$ with norm $A_{q}>0$. Assume that $\vec{K}$ satisfies Hörmander's conditions (1.8) and (1.9) for some $C_{H}>0$. Then there exist positive constants $C_{\mathscr{C}}$, $\widetilde{C}_{\mathscr{X}}$ depending only on $\mathscr{X}$ such that for all $\mathscr{B}_{1}$-valued functions $\left\{F_{j}\right\}_{j \in \mathrm{N}}$,

$$
\begin{aligned}
\left\|\left(\sum_{j \in \mathrm{N}}\left\|\vec{T}\left(F_{j}\right)\right\|_{\mathscr{B}_{2}}^{q}\right)^{1 / q}\right\|_{L^{1, \infty}\left(\mathscr{X}, \mathscr{B}_{2}\right)} & \\
\leq \widetilde{C}_{\mathscr{X}}\left(C_{H}+A_{q}\right) \| & \left(\sum_{j \in \mathrm{N}}\left\|F_{j}\right\|_{\mathscr{B}_{1}}^{q}\right)^{1 / q} \|_{L^{1}\left(\mathscr{X}, \mathscr{B}_{1}\right)}
\end{aligned}
$$

and

$$
\begin{aligned}
&\left\|\left(\sum_{j \in \mathrm{N}}\left\|\vec{T}\left(F_{j}\right)\right\|_{\mathscr{B}_{2}}^{q}\right)^{1 / q}\right\|_{L^{p}\left(\mathscr{O}, \mathscr{B}_{2}\right)} \\
& \quad \leq C_{\mathscr{X}} C_{p}\left(C_{H}+A_{q}\right)\left\|\left(\sum_{j \in \mathrm{N}}\left\|F_{j}\right\|_{\mathscr{B}_{1}}^{q}\right)^{1 / q}\right\|_{L^{p}\left(\mathscr{X}, \mathscr{B}_{1}\right)},
\end{aligned}
$$

where $C_{p}=\max \left\{p,(p-1)^{-1}\right\}$.

\section{Proof of Theorem 1.2}

To prove the Fefferman-Stein vector-valued maximal function inequality, the existence of the following approximation of the identity on spaces of homogeneous type, proved in [9, Theorem 2.1], plays a key role. 
LEMMA 3.1. There exist $C_{3}>1$ and a sequence of bounded linear integral operators $\left\{S_{k}\right\}_{k \in \mathrm{Z}}$ on $L^{2}(\mathscr{X})$ such that for all $k \in \mathrm{Z}$ and all $x, x^{\prime}, y$ and $y^{\prime} \in \mathscr{X}$, $S_{k}(x, y)$, the integral kernel of $S_{k}$ is a measurable function from $\mathscr{X} \times \mathscr{X}$ into $\mathrm{R}^{+}$satisfying

(i) $S_{k}(x, y)=0$ if $d(x, y)>2^{2-k}$ and $S_{k}(x, y) \leq C_{3} \frac{1}{V_{2-k}(x)+V_{2-k}(y)}$;

(ii) $S_{k}(x, y)=S_{k}(y, x)$;

(iii) $\left|S_{k}(x, y)-S_{k}\left(x^{\prime}, y\right)\right| \leq C_{3} 2^{k} d\left(x, x^{\prime}\right) \frac{1}{V_{2-k}(x)+V_{2-k}(y)}$ for $d\left(x, x^{\prime}\right) \leq 2^{3-k}$;

(iv) $\left|\left[S_{k}(x, y)-S_{k}\left(x, y^{\prime}\right)\right]-\left[S_{k}\left(x^{\prime}, y\right)-S_{k}\left(x^{\prime}, y^{\prime}\right)\right]\right| \leq C_{3} 2^{2 k} \frac{d\left(x, x^{\prime}\right) d\left(y, y^{\prime}\right)}{V_{2-k}(x)+V_{2-k}(y)}$ for $d\left(x, x^{\prime}\right) \leq 2^{3-k}$ and $d\left(y, y^{\prime}\right) \leq 2^{3-k}$;

(v) $\int_{\mathscr{X}} S_{k}(x, y) d \mu(x)=1$;

(vi) $C_{3} V_{2^{-k}}(x) S_{k}(x, x) \geq 1$.

REMARK 3.2. From (iii) and (vi) above, we deduce that for any $C_{4} \in$ $\left(0,\left(C_{3}\right)^{-2}\right)$, there exists $C_{5}>0$ such that for any $k \in \mathrm{Z}$ and $x, y \in \mathscr{X}$ satisfying $d(x, y)<C_{4} 2^{-k}$,

$$
C_{5} V_{2^{-k}}(x) S_{k}(x, y)>1,
$$

where $C_{5}=C_{3} /\left(1-\left(C_{3}\right)^{2} C_{4}\right)$.

Proof of Theorem 1.2. If $q=\infty$, then (1.12) and (1.13) can be deduced directly from the fact $\sup _{j \in \mathrm{N}} \mathcal{M}\left(f_{j}\right)(x) \leq \mathcal{M}\left(\sup _{j \in \mathrm{N}}\left|f_{j}\right|\right)(x)$ and the boundedness of $\mathscr{M}$. Thus we only need to consider the case $q<\infty$.

Let $\left\{S_{k}\right\}_{k \in \mathrm{Z}}$ be as in Lemma 3.1. For $f \in L_{\mathrm{loc}}^{1}(\mathscr{X})$, set

$$
\mathscr{M}_{0}(f)(x)=\sup _{k \in Z}\left|S_{k}(f)(x)\right| \text {. }
$$

Notice that for any $x \in \mathscr{X}$, by (1.2),

$$
\begin{aligned}
\mathscr{M}(f)(x) & =\sup _{r>0} \frac{1}{\mu(B(x, r))} \int_{B(x, r)}|f(y)| d \mu(y) \\
& \sim \sup _{k \in \mathrm{Z}} \frac{1}{V_{2^{-k}}(x)} \int_{B\left(x, 2^{-k}\right)}|f(y)| d \mu(y),
\end{aligned}
$$

which together with (3.1) yields that for any $x \in \mathscr{X}$,

$$
\mathcal{M}(f)(x) \lesssim \sup _{k \in Z} \int_{B\left(x, 2^{-k}\right)} S_{k}(x, y)|f(y)| d \mu(y) \lesssim \mathcal{M}_{0}(|f|)(x) .
$$


We will obtain the claimed vector-valued inequality for "bigger" maximal operator $\mathscr{M}_{0}$. For any given $y, z \in \mathscr{X}$ and $y \neq z$, set $J_{1}=\{k \in Z: d(y, z) \leq$ $\left.2^{3-k}\right\}$ and $J_{2}=\mathrm{Z} \backslash J_{1}$. Then we write,

$$
\begin{aligned}
\int_{d(x, y)} \geq 2 d(y, z) & \sup _{k \in Z}\left|S_{k}(x, y)-S_{k}(x, z)\right| d \mu(x) \\
& \leq \sum_{k \in Z} \int_{d(x, y) \geq 2 d(y, z)}\left|S_{k}(x, y)-S_{k}(x, z)\right| d \mu(x) \\
& \equiv \sum_{k \in Z} \mathrm{I}_{k} .
\end{aligned}
$$

For each $k \in J_{2}$, by Lemma 3.1 (i) and the triangle inequality for the metric $d$, we can deduce that $\mathrm{I}_{k}=0$. For any $k \in J_{1}$, set

$$
D_{k}=\{x \in \mathscr{X}: d(x, y) \geq 2 d(y, z)\} \bigcap\left(B\left(y, 2^{2-k}\right) \bigcup B\left(z, 2^{2-k}\right)\right) .
$$

Thus when $k \in J_{1}$, by the support condition of $S_{k}$ and the regularity of $S_{k}$,

$$
\begin{aligned}
\mathrm{I}_{k} & =\int_{D_{k}}\left|S_{k}(x, y)-S_{k}(x, z)\right| d \mu(x) \\
& \lesssim \int_{D_{k}} \frac{2^{k} d(y, z)}{V_{2^{-k}}(x)+V_{2^{-k}}(y)} d \mu(x) \lesssim 2^{k} d(y, z),
\end{aligned}
$$

which implies that $\sum_{k \in J_{1}} \mathrm{I}_{k} \lesssim 1$. Combining this with (3.3) yields that

$$
\sup _{y, z \in \mathscr{X}} \int_{d(x, y) \geq 2 d(y, z)} \sup _{k \in Z}\left|S_{k}(x, y)-S_{k}(x, z)\right| d \mu(x) \lesssim 1 .
$$

Set $\mathscr{B}_{1}=\mathrm{C}, \mathscr{B}_{2}=\ell^{\infty}$ and view $\mathscr{M}_{0}$ as the linear operator $f \rightarrow\left\{S_{k}(f)\right\}_{k \in \mathrm{Z}}$ that maps $\mathscr{B}_{1}$-valued functions to $\mathscr{B}_{2}$-valued functions. Precisely, we define a $\mathscr{B}_{2}$-valued kernel $\vec{K}(x, y)=\left\{S_{k}(x, y)\right\}_{k \in z}$ and a $\mathscr{B}_{2}$-valued linear operator $\vec{M}_{0}(f)=\left\{S_{k}(f)\right\}_{k \in Z}$. For any $f \in L^{\infty}(\mathscr{X})$ and $x \in \mathscr{X}$,

$$
\left|S_{k}(f)(x)\right| \leq \int_{d(x, y) \leq 2^{2-k}} S_{k}(x, y)|f(y)| d \mu(y) \lesssim\|f\|_{L^{\infty}(\mathscr{X})} .
$$

Therefore, $\vec{M}_{0}$ is bounded from $L^{\infty}\left(\mathscr{X}, \mathscr{B}_{1}\right)$ to $L^{\infty}\left(\mathscr{X}, \mathscr{B}_{2}\right)$. By (3.4) and the symmetry of $S_{k}(x, y)$, we have that $\vec{K}$ satisfies Hörmander's conditions (1.8) and (1.9). Then applying Theorem 1.1 yields that for any $q \in(1, \infty)$,

$$
\left.\left\|\overrightarrow{\mathscr{M}}_{0}(f)\right\|_{L^{q}\left(\mathscr{X}, \mathscr{B}_{2}\right)} \lesssim \max \left\{1,(q-1)^{-1}\right)\right\}\|f\|_{L^{q}\left(\mathscr{X}, \mathscr{B}_{1}\right)} .
$$


Furthermore, using Corollary 2.9, we obtain

$$
\begin{aligned}
& \left\|\left(\sum_{j \in \mathrm{N}}\left\|\overrightarrow{\mathcal{M}}_{0}\left(f_{j}\right)\right\|_{\mathscr{B}_{2}}^{q}\right)^{1 / q}\right\|_{L^{1, \infty}\left(\mathscr{L}, \mathscr{B}_{2}\right)} \\
& \lesssim \max \left\{1,(q-1)^{-1}\right\}\left\|\left(\sum_{j \in \mathrm{N}}\left\|f_{j}\right\|_{\mathscr{B}_{1}}^{q}\right)^{1 / q}\right\|_{L^{1}\left(\mathscr{L}, \mathscr{B}_{1}\right)}
\end{aligned}
$$

and

$$
\left\|\left(\sum_{j \in \mathrm{N}}\left\|\overrightarrow{\mathscr{M}}_{0}\left(f_{j}\right)\right\|_{\mathscr{B}_{2}}^{q}\right)^{1 / q}\right\|_{L^{p}\left(\mathscr{X}, \mathscr{B}_{2}\right)} \lesssim C_{p, q}\left\|\left(\sum_{j \in \mathrm{N}}\left\|f_{j}\right\|_{\mathscr{B}_{1}}^{q}\right)^{1 / q}\right\|_{L^{p}\left(\mathscr{\mathscr { L }}, \mathscr{B}_{1}\right)},
$$

where $C_{p, q}=\max \left\{p,(p-1)^{-1}\right\} \max \left\{1,(q-1)^{-1}\right\}$. Combining this with (3.2) and using the fact $\left\|\overrightarrow{\mathscr{M}}_{0}\left(f_{j}\right)(x)\right\|_{\mathscr{B}_{2}}=\mathscr{M}_{0}\left(f_{j}\right)(x)$, we deduce the desired inequalities and complete the proof of Theorem 1.2.

Remark 3.3. Let $d$ be a quasi-metric, which means that there exists $A_{0} \geq 1$ such that for all $x, y, z \in \mathscr{X}, d(x, y) \leq A_{0}(d(x, z)+d(z, y))$. Recall that Macías and Segovia [11, Theorem 2] proved that there exists an equivalent quasi-metric $\widetilde{d}$ such that all balls corresponding to $\widetilde{d}$ are open in the topology induced by $\widetilde{d}$, and there exist constants $A_{0}^{\prime}>0$ and $\theta \in(0,1)$ such that for all $x, y, z \in \mathscr{X}$,

$$
|\widetilde{d}(x, z)-\widetilde{d}(y, z)| \leq A_{0}^{\prime} \widetilde{d}(x, y)^{\theta}[\widetilde{d}(x, z)+\widetilde{d}(y, z)]^{1-\theta} .
$$

It is known that the approximation of the identity as in Lemma 3.1 also exists for $\widetilde{d}$; see [9]. Obviously, all results in this paper are invariant on equivalent quasi-metrics. From these facts, we deduce that all conclusions of this paper are still valid for quasi-metrics.

\section{REFERENCES}

1. Benedek, A., Calderón, A.-P., and Panzone, R., Convolution operators on Banach space valued functions, Proc. Nat. Acad. Sci. U.S.A. 48 (1962), 356-365.

2. Coifman, R. R., and Weiss, G., Analyse harmonique non-commutative sur certains espaces homogènes, Lecture Notes in Math. 242, Springer, Berlin 1971.

3. Coifman, R. R., and Weiss, G., Extensions of Hardy spaces and their use in analysis, Bull. Amer. Math. Soc. 83 (1977), 569-645.

4. Diestel, J., and Uhl Jr., J. J., Vector Measures, Mathematical Surveys 15, American Mathematical Society, Providence, R.I. 1977.

5. Fefferman C., and Stein, E. M., Some maximal inequalities, Amer. J. Math. 93 (1971), 107116.

6. García-Cuerva, J., and Rubio de Francia, J. L., Weighted Norm Inequalities and Related Topics, North-Holland Math. Studies 116, North-Holland, Amsterdam 1985. 
7. Grafakos, L., Classical and Modern Fourier Analysis, Prentice Hall, Upper Saddle River, N.J. 2004.

8. Han, Y., Müller, D., and Yang, D., Littlewood-Paley characterizations for Hardy spaces on spaces of homogeneous type, Math. Nachr. 279 (2006), 1505-1537.

9. Han, Y., Müller, D., and Yang, D., A theory of Besov and Triebel-Lizorkin spaces on metric measure spaces modeled on Carnot-Carathéodory spaces, Abstr. Appl. Anal. 2008, Art. ID 893409 (to appear).

10. Heinonen, J., Lectures on Analysis on Metric Spaces, Universitext, Springer, New York 2001.

11. Macías, R. A., and Segovia, C., Lipschitz functions on spaces of homogeneous type, Adv. in Math. 33 (1979), 257-270.

12. Müller, D., and Yang, D., A difference characterization of Besov and Triebel-Lizorkin spaces on RD-spaces, Forum Math. (to appear).

13. Nagel, A., and Stein, E. M., On the product theory of singular integrals, Rev. Mat. Iberoamericana 20 (2004), 531-561.

14. Stein, E. M., Harmonic Analysis: Real-variable Methods, Orthogonality, and Oscillatory Integrals, Princeton Mathematical Series 43, Princeton University Press, Princeton, N.J., 1993.

15. Stein, E. M., and Weiss, G., Introduction to Fourier Analysis on Euclidean Spaces, Princeton Mathematical Series 32, Princeton University Press, Princeton, N.J. 1971.

16. Strömberg, J.-O., and Torchinsky, A., Weighted Hardy Spaces, Lecture Notes in Math. 1381, Springer, Berlin 1989.

17. Yosida, K., Functional Analysis, Classics in Mathematics, Springer, Berlin 1995.

LOUKAS GARAFAKOS

DEPARTMENT OF MATHEMATICS

UNIVERSITY OF MISSOURI

COLUMBIA, MO 65211

USA

E-mail: loukas@math.missouri.edu
LIGUANG LIU \& DACHUN YANG

SCHOOL OF MATHEMATICAL SCIENCES

BEIJING NORMAL UNIVERSITY

LABORATORY OF MATHEMATICS AND COMPLEX SYSTEMS

MINISTRY OF EDUCATION

BEIJING 100875

THE PEOPLE'S REPUBLIC OF CHINA

E-mail: liuliguang@mail.bnu.edu.cn, dcyang@bnu.edu.cn 